\title{
Microencapsulation of fish oil using Hydroxypropyl methylcellulose as a carrier material by spray drying
}

\begin{abstract}
Spray drying is an important method in the food industry for the production of encapsulated oil to improve the handling and flow properties of the powder. In this study, the effect of mixture of polymers on the encapsulation of fish oil by spray drying was investigated. Fish oil powder were produced using different ratios of mixtures of hydroxypropyl methylcellulose (HPMC) 15 cps and HPMC 5 cps. Scanning electron microscopy and the amount of extracted oil from the surface revealed that the formulation containing high concentration of polymer mixture provided the highest protective and prolonged effect on the covering of fish oil. The particle sizes of less than $60 \mu \mathrm{m}$ were obtained for all the formulations. The powder density was very suitable, which improves the flowability of the powder. Microencapsulation efficiency (69.16-74.75\%) and surface morphology of encapsulated oil showed that the stability was increased and hence increased its acceptability as alternative primary polymers.
\end{abstract}

